This item was submitted to Loughborough's Research Repository by the author.

Items in Figshare are protected by copyright, with all rights reserved, unless otherwise indicated.

\title{
Trapped modes for off-centre structures in guides
}

PLEASE CITE THE PUBLISHED VERSION

LICENCE

CC BY-NC-ND 4.0

\section{REPOSITORY RECORD}

Linton, C.M., M. Mclver, P. Mclver, Keith Ratcliffe, and J. Zhang. 2019. "Trapped Modes for Off-centre Structures in Guides". figshare. https://hdl.handle.net/2134/688. 


\title{
Trapped modes for off-centre structures in guides
}

\author{
by \\ C M Linton, M McIver, P McIver, K Ratcliffe and J Zhang \\ Department of Mathematical Sciences, Loughborough University, \\ Leicestershire LE11 3TU
}

\begin{abstract}
The existence of trapped modes near obstacles in two-dimensional waveguides is well established when the centre-line of the guide is a line of symmetry for the geometry. In this paper we examine cases where no such line of symmetry exists. The boundary condition on the obstacle is of Neumann type and both Neumann and Dirichlet conditions on the guide walls are treated. A variety of techniques (variational methods, boundary integral equations, slender-body theory, modified residue calculus theory) are used to investigate trapped mode phenomena in a number of different frequency bands.
\end{abstract}

\section{Introduction}

Symmetry plays a key role in determining the existence or otherwise of trapped modes (acoustic resonances) near obstacles in waveguides. Such modes represent oscillations of finite energy, localized about the obstacle, and in the case of a sound-hard obstacle situated in a sound-hard two-dimensional parallel-plate guide so that the resulting geometry is symmetric about the centreline, the existence of trapped modes was proved by Evans, Levitin, and Vassiliev (1994). The symmetry of the geometry makes it possible to decompose the problem into two parts, one symmetric and the other antisymmetric about the centreline. The antisymmetric problem exhibits a cut-off frequency, below which waves cannot propagate down the guide (i.e. the continuous spectrum is bounded away from zero) and a variational technique can be used to prove that trapped modes (corresponding to eigenvectors of the differential operator) exist below the cut-off.

In the problem discussed above, the boundary condition for the potential on the guide walls is that the normal derivative vanishes and thus the walls can equally well be thought of as representing lines of symmetry. Hence the trapped modes also exist in the presence of an infinite array of symmetric obstacles. For such an array we could look for modes which are antisymmetric, rather than symmetric, about the lines mid-way between the obstacles. Again we can use the symmetry of the geometry to decompose the problem into two parts, one symmetric and the other antisymmetric about the centreline, and in this case both problems exhibit cut-offs below which propagating modes cannot exist. However, for the case of symmetry about the centreline trapped modes are known not to exist below the cut-off frequency for a wide class of geometries (McIver and Linton 1995) and for the antisymmetric problem a variational argument can be used to show that modes do exist provided the obstacle satisfies a simple geometric condition (Evans and Porter 1998).

In this paper we investigate the situation where the geometry is not symmetric about the centreline of the guide. Thus we consider a sound-hard obstacle placed within a 


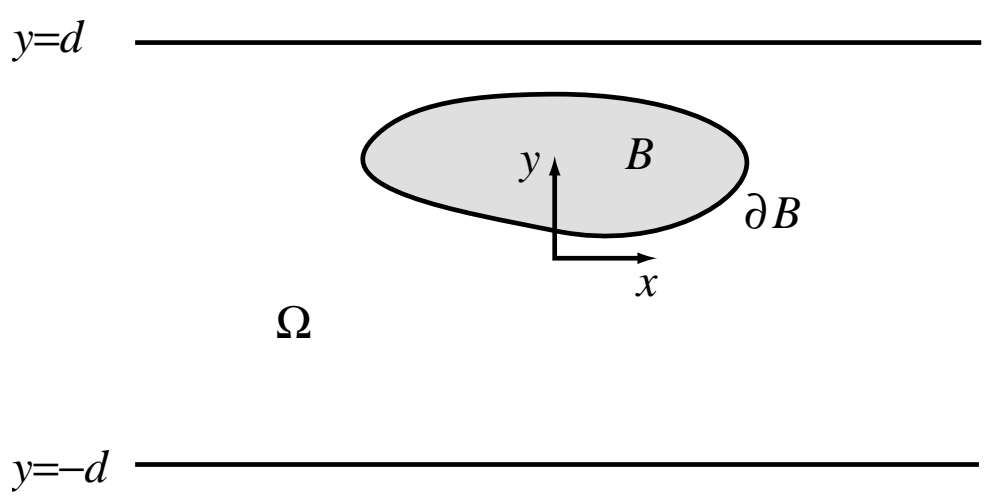

Figure 1: Definition sketch.

parallel-plate waveguide and we treat both Neumann and Dirichlet boundary conditions on the guide walls. Thus the problems under consideration can, by reflection, be extended to problems involving infinite arrays of obstacles. A number of different techniques are employed to shed light on the existence of trapped modes in various different situations; the emphasis is on the results rather than the methods.

All the problems which are considered in this paper are of the form (see Figure 1)

$$
\begin{aligned}
\left(\nabla^{2}+k^{2}\right) \phi & =0 \quad \text { in } \quad \Omega, \\
\frac{\partial \phi}{\partial n} & =0 \quad \text { on } \quad \partial B, \\
\phi & \rightarrow 0 \quad \text { as } \quad|x| \rightarrow \infty
\end{aligned}
$$

together with either

$$
\phi=0 \quad \text { on } \quad y= \pm d
$$

or

$$
\frac{\partial \phi}{\partial y}=0 \quad \text { on } \quad y= \pm d
$$

The condition (1.3) is equivalent, in the context of this problem, to the assertion that $\phi$ represents an oscillation of finite energy, i.e. $\int_{\Omega}|\nabla \phi|^{2} \mathrm{~d} \Omega<\infty$. The aim will be to find values of the parameter $k$ (related to the angular frequency of oscillations $\omega$ and the speed of sound $c$ through $k=\omega / c$ ) for which the problem (1.1)-(1.3) with either (1.4) or (1.5) possesses non-trivial solutions. These will be referred to as the Dirichlet and Neumann problems respectively and any non-trivial potentials $\phi$ which satisfy these problems are called trapped modes.

Separation of variables shows that solutions in an empty guide take the form

$$
\sin \lambda_{n}(y+d) \mathrm{e}^{ \pm \gamma_{n} x}, \quad n=1,2, \ldots,
$$

for the Dirichlet problem and

$$
\cos \lambda_{n}(y+d) \mathrm{e}^{ \pm \gamma_{n} x}, \quad n=0,1, \ldots,
$$


for the Neumann problem, where

$$
\lambda_{n}=n \pi / 2 d \quad \text { and } \quad \gamma_{n}=\left(\lambda_{n}^{2}-k^{2}\right)^{1 / 2}=-\mathrm{i}\left(k^{2}-\lambda_{n}^{2}\right)^{1 / 2} .
$$

If $\gamma_{n}$ is imaginary these correspond, in the context of the velocity potential

$$
\Phi(x, y, t)=\operatorname{Re}\left[\phi(x, y) \mathrm{e}^{-\mathrm{i} \omega t}\right]
$$

to propagating modes, whereas if $\gamma_{n}$ is real we only consider modes which decay down the guide. Hence the number of possible propagating modes in the guide depends on the value of $k$. If $\lambda_{N}<k<\lambda_{N+1}$ there are $N$ modes possible for the Dirichlet problem and $N+1$ possible modes for the Neumann problem.

In the following section we consider the case of the Dirichlet problem for $k<\lambda_{1}$. This is a situation where the frequency is below the cut-off for propagating modes and so standard variational methods are applicable. The interesting thing about this situation is that for a large class of obstacles trapped modes are known not to exist, but we show that provided the obstacle is sufficiently far away from the centreline modes do exist. This existence result is confirmed by numerical calculations for circular shapes and is also consistent with results found using a slender-body theory based on that developed in Evans and McIver (1991). For example, an obstacle geometry might be specified using two parameters $a$ and $b$ that describe, respectively, the size and position within the guide (there are then two non-dimensional parameters $a / d$ and $b / d$ ). In general, obstacles that support trapped modes are found within two-dimensional regions of the $(a / d, b / d)$ parameter space. For the special case of a thin plate aligned with the guide walls, it turns out that modes are possible for all positions of the plate (except on the centreline), and calculations for this geometry are given based on modified residue calculus theory.

In section 3 we examine the Neumann problem, again for $k<\lambda_{1}$. In this case one propagating mode is possible and the existence of trapped modes is much harder to establish. Numerical calculations based on an integral equation formulation are presented which provide strong numerical evidence for existence and which are consistent with the results from slender-body theory. The existence of the possible propagating mode leads to the need to satisfy a side condition which corresponds to forcing the amplitude of this mode to be zero. In order to be able to do this, we need to introduce an extra geometrical parameter into the definition of the obstacle. For example, an obstacle geometry might be defined in terms of the parameters $a / d$ and $b / d$ as above, and a further non-dimensional parameter $\delta$ describing the shape of the obstacle. In general, obstacles that support trapped modes are found on two-dimensional surfaces in the $(a / d, b / d, \delta)$ parameter space.

One propagating mode is also possible for the Dirichlet problem if $\lambda_{1}<k<\lambda_{2}$ and this situation is considered in section 4 . Slender-body theory suggests that trapped modes can be found in this case also. The only known example of a trapped mode of this type is that computed for a semicircle with a specific radius, attached to one of the walls, in Evans and Porter (1998) and the numerical value for the radius predicted by the slender-body theory is in good agreement with Evans and Porter's result.

It is possible to increase $k$ systematically through the cut-off values $\lambda_{n}$ and for each extra propagating mode that is introduced, an extra side condition needs to be satisfied. Thus it is anticipated that in order to find trapped modes for higher frequencies one would need to consider obstacles defined by more parameters. We have not followed this tack here. Rather, in section 5, we consider a specific example of the Neumann problem for the frequency range $\lambda_{2}<k<\lambda_{3}$. The geometry is that of a thin plate aligned with the guide 
walls, for which atypical results are to be expected (see Evans, Linton, and Ursell (1993) and section 2 below). The given frequency range allows for three propagating modes in the guide and, provided the plate is not too far from the centreline, two modes in the region between the plate and each wall. Residue calculus theory is used to show that in this case trapped modes exist for a discrete set of points in the $(a / d, b / d)$ parameter space.

\section{$2 \quad$ Dirichlet modes $k d<\pi / 2$}

In this section we consider the Dirichlet problem with $k<\lambda_{1}$, a condition which prohibits the existence of modes propagating down the guide since $\gamma_{n}$ is real for all $n \geq 1$. It is known that for some shapes $B$ trapped modes do not exist. For example, McIver and Linton (1995) showed that if the normal $\left(n_{x}, n_{y}\right)$ to the boundary $\partial B$, directed out of the fluid region, satisfies

$$
\begin{array}{ll}
n_{y} \leq 0 & \text { if } \quad y \geq 0 \\
n_{y} \geq 0 & \text { if } \quad y \leq 0
\end{array}
$$

then no trapped modes exist for $k d<\pi / 2$. Shapes which satisfy this condition include, for example, rectangles which enclose the origin with sides parallel to the guide walls, or any number of circles with centres on the guide centreline.

\section{Variational principle}

The existence of modes for some geometries can be proved using a standard variational argument. Suppose that $\partial B$ can be defined by the union of the two curves

$$
\begin{gathered}
y=y_{+}(x), \quad y=y_{-}(x), \quad x \in[-a, a], \\
y_{+}( \pm a)=y_{-}( \pm a), \quad-d<y_{-}(x) \leq y_{+}(x)<d .
\end{gathered}
$$

The trial function

$$
\psi=\left\{\begin{array}{lll}
\cos \left(\lambda_{1} y\right) & \text { if } & |x|<a \\
\cos \left(\lambda_{1} y\right) \exp (-\epsilon(|x|-a) / a) & \text { if } & |x|>a
\end{array}\right.
$$

where $\epsilon>0$, satisfies (1.3) and (1.4), is continuous across $x= \pm a$, and has the property that as $\epsilon \rightarrow 0$,

$$
\frac{\int_{\Omega}(\nabla \psi)^{2} \mathrm{~d} \Omega}{\int_{\Omega} \psi^{2} \mathrm{~d} \Omega}=\lambda_{1}^{2}\left(1+\frac{2 \epsilon}{\pi a} \int_{-a}^{a} \sin \lambda_{1}\left(y_{+}-y_{-}\right) \cos \lambda_{1}\left(y_{+}+y_{-}\right) \mathrm{d} x\right)+O\left(\epsilon^{2}\right) .
$$

It follows that the lowest point of the spectrum (the spectral parameter being $k^{2}$ ) of the negative Laplacian on $\Omega$ with Dirichlet conditions on the guide walls but a Neumann condition on $\partial B$ is less than $\lambda_{1}^{2}=\pi^{2} / 4 d^{2}$, provided

$$
\int_{-a}^{a} \sin \lambda_{1}\left(y_{+}-y_{-}\right) \cos \lambda_{1}\left(y_{+}+y_{-}\right) \mathrm{d} x<0 .
$$

As long as the possibility that $y_{+}(x)$ and $y_{-}(x)$ are identical is excluded, in which case $B$ would be an obstacle of zero thickness, we have

$$
\sin \lambda_{1}\left(y_{+}-y_{-}\right)>0
$$


since $0 \leq y_{+}(x)-y_{-}(x) \leq 2 d$ for all $x \in[-a, a]$. Thus a sufficient condition for the existence of a trapped mode is

$$
\left|y_{+}+y_{-}\right|>d \quad \text { for all } x \in[-a, a]
$$

For the special case where $y_{+}(x)=b+f(x)$ and $y_{-}(x)=b-f(x),(f(x) \geq 0,|b \pm f(x)|<d)$, in which case we are dealing with an obstacle with a line of symmetry parallel to the guide walls, the condition reduces to

$$
|b| / d>1 / 2 .
$$

\section{Numerical computations}

The condition (2.7) is consistent with the numerical results for a circle with radius $a / d=$ 0.2 and centre at $y=b$, presented in Figure 2 and computed using the integral equation method described in McIver et al. (2001). However, the results show that (2.7) is not a necessary condition for the existence of trapped modes. Further numerical results (not shown) suggest that the largest circle for which this type of trapped mode exists has $a / d \approx 0.525$ and $b / d \approx 0.475$. When $b / d=0.8$, for the example given, the circle is touching the guide wall, but the numerical calculations can be continued beyond this, corresponding to the case where there is a protrusion into the guide. When this protrusion becomes a semicircle (i.e. when $b / d=1$ ) the situation is equivalent to a circle on the centreline of a channel of width $4 d$ with a Dirichlet condition imposed on the centreline exterior to the circle. For this case Porter and Evans (1999) proved the existence of a mode provided $J_{1}(\pi a / d)>0$ (i.e. $\left.a / d \lesssim 1.22\right)$ and Maniar and Newman (1997) computed modes for $a / d \lesssim 1.35$.

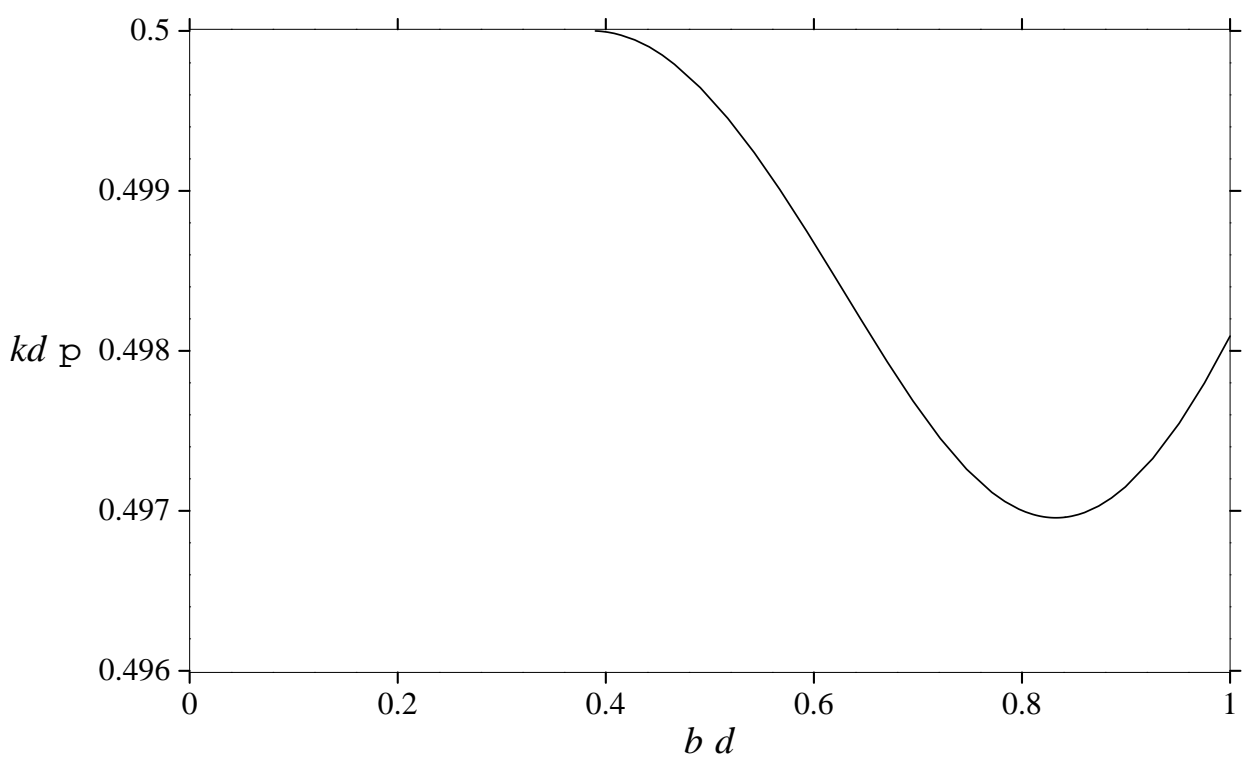

Figure 2: Trapped-mode wavenumbers $k d / \pi$ plotted against $b / d$ for a circle with when $a / d=0.2$. 


\section{Slender shapes}

Further insight can be gained by considering bodies which are slender. For convenience we restrict attention to bodies symmetric in $x$ and whose boundary in $x \geq 0$ is described by

$$
x=\epsilon f(y), \quad y \in(-d, d), \quad \text { where } \quad \epsilon \ll 1 .
$$

A normal vector to the body surface is $\left(n_{x}, n_{y}\right)=\left(1,-\epsilon f^{\prime}(y)\right)$ and a slender body is defined to be one for which

$$
f^{\prime}(y)=O(1) \quad \text { as } \quad \epsilon \rightarrow 0,
$$

so that $n_{y}=O(\epsilon)$. The method described in section 4 of McIver et al. (2001) and in McIver (2001) shows that in the limit as $\epsilon \rightarrow 0$, trapped modes exist provided

$$
\gamma_{1}=\frac{\epsilon \pi}{4 d^{2}} \int_{-d}^{d} f^{\prime}(y) \sin 2 \lambda_{1} y \mathrm{~d} y
$$

Since $\gamma_{1}$ is by definition positive for the range of values of $k$ being considered, the righthand side must be positive for this equation to have any solutions for $k$.

Consider a shape defined by a function $f(y)$ which is zero if $y \notin(b-c, b+c),(|b \pm c|<d)$, and which satisfies

$$
f^{\prime}(b-u)=-f^{\prime}(b+u), \quad f^{\prime}(b+u)<0, \quad \text { for } \quad 0<u<c .
$$

Then the integral on the right-hand side of (2.10) reduces to

$$
\int_{0}^{c} f^{\prime}(b+u) \cos 2 \lambda_{1} b \sin 2 \lambda_{1} u \mathrm{~d} u
$$

and in order for this to be positive we must have $b / d>1 / 2$, which is consistent with the variational proof and the numerical results given above.

\section{Thin plates}

The slender body theory does not apply when $B$ has finite extent in the $x$-direction but zero thickness, and the particular test function used in the variational proof above yields no information. However, the case of a thin plate aligned with the guide walls can be analyzed using the so-called modified residue calculus theory. This was done for an offcentre plate in a guide with Neumann conditions on the walls (and $k d<\pi / 2$ ) in Evans, Linton, and Ursell (1993), though in the problem under consideration here the situation is somewhat simpler as the frequency is below the first cut-off for propagating modes. If we consider a plate of length $2 a$ situated at $y=b$, then (1.2) becomes

$$
\frac{\partial \phi}{\partial y}=0 \quad \text { on } \quad y=b,-a<x<a
$$

and the boundedness of $\phi$ at the ends of the plate leads to the edge conditions

$$
\nabla \phi=O\left(r_{ \pm}^{-1 / 2}\right) \quad \text { as } \quad r_{ \pm} \equiv\left\{(x \mp a)^{2}+(y-b)^{2}\right\}^{1 / 2} \rightarrow 0 .
$$


The results of applying the modified residue calculus technique to this problem are as follows. Without loss of generality we will assume that $b>0$ and then define

$$
\begin{array}{cc}
\nu_{n}=\frac{\left(n-\frac{1}{2}\right) \pi}{d-b}, & \mu_{n}=\frac{\left(n+\frac{1}{2}\right) \pi}{d+b}, \\
\alpha_{n}=\left(\nu_{n}^{2}-k^{2}\right)^{1 / 2}, & \beta_{n}=\left(\mu_{n}^{2}-k^{2}\right)^{1 / 2} .
\end{array}
$$

It then follows that if

$$
\frac{\pi d}{d+b}<2 k d<\pi
$$

then $\alpha_{n}, \beta_{n}, \gamma_{n}, n \geq 1$ are all real, whereas

$$
\beta_{0}=-\mathrm{i} \beta^{\prime}, \quad \text { where } \quad \beta^{\prime}=\left(k^{2}-\mu_{0}^{2}\right)^{1 / 2},
$$

is imaginary. The condition for the existence of trapped modes symmetric about $x=0$ is

$$
\beta^{\prime}(a+\Theta)=\chi+\delta+\left(n+\frac{1}{2}\right) \pi
$$

for some integer $n$, where

$$
\begin{aligned}
\chi & =\sum_{n=1}^{\infty}\left(\tan ^{-1} \frac{\beta^{\prime}}{\alpha_{n}}+\tan ^{-1} \frac{\beta^{\prime}}{\beta_{n}}-\tan ^{-1} \frac{\beta^{\prime}}{\gamma_{n}}\right), \\
\delta & =\arg \left(1-\sum_{n=1}^{\infty}\left[\frac{A_{n}}{\alpha_{n}+\mathrm{i} \beta^{\prime}}+\frac{B_{n}}{\beta_{n}+\mathrm{i} \beta^{\prime}}\right]\right) \\
\Theta & =\frac{1}{\pi}\left((d+b) \ln \frac{2 d}{d+b}+(d-b) \ln \frac{2 d}{d-b}\right) .
\end{aligned}
$$

Here $A_{n}$ and $B_{n}$ are the solutions to the infinite system of coupled equations

$$
\begin{aligned}
& A_{m}+C_{m} \sum_{n=1}^{\infty}\left(\frac{A_{n}}{\alpha_{m}+\alpha_{n}}+\frac{B_{n}}{\alpha_{m}+\beta_{n}}\right)=C_{m} \\
& B_{m}+D_{m} \sum_{n=1}^{\infty}\left(\frac{A_{n}}{\beta_{m}+\alpha_{n}}+\frac{B_{n}}{\beta_{m}+\beta_{n}}\right)=D_{m}
\end{aligned}
$$

where $m=1,2, \ldots$, and

$$
\begin{aligned}
C_{m} & =\frac{2 \alpha_{m}\left(\gamma_{m}-\alpha_{m}\right)\left(\beta_{m}+\alpha_{m}\right)}{\left(\gamma_{m}+\alpha_{m}\right)\left(\beta_{m}-\alpha_{m}\right)} \mathrm{e}^{-2 \alpha_{m}(a+\Theta)} \prod_{\substack{n=1 \\
n \neq m}}^{\infty} \frac{\left(1+\alpha_{m} / \alpha_{n}\right)\left(1+\alpha_{m} / \beta_{n}\right)\left(1-\alpha_{m} / \gamma_{n}\right)}{\left(1-\alpha_{m} / \alpha_{n}\right)\left(1-\alpha_{m} / \beta_{n}\right)\left(1+\alpha_{m} / \gamma_{n}\right)} \\
D_{m} & =\frac{2 \beta_{m}\left(\gamma_{m}-\beta_{m}\right)\left(\alpha_{m}+\beta_{m}\right)}{\left(\gamma_{m}+\beta_{m}\right)\left(\alpha_{m}-\beta_{m}\right)} \mathrm{e}^{-2 \beta_{m}(a+\Theta)} \prod_{\substack{n=1 \\
n \neq m}}^{\infty} \frac{\left(1+\beta_{m} / \alpha_{n}\right)\left(1+\beta_{m} / \beta_{n}\right)\left(1-\beta_{m} / \gamma_{n}\right)}{\left(1-\beta_{m} / \alpha_{n}\right)\left(1-\beta_{m} / \beta_{n}\right)\left(1+\beta_{m} / \gamma_{n}\right)}
\end{aligned}
$$

Because of the presence of the exponentially decaying factors in (2.24) and (2.25), the system of equations $(2.22),(2.23)$ converges very rapidly and provides an extremely efficient method for computing the unknowns $A_{m}$ and $B_{m}$.

For the case of antisymmetry about $x=0$, the condition for the existence of trapped modes is

$$
\beta^{\prime}(a+\Theta)=\chi+\delta+n \pi
$$


for some integer $n$, where $\Theta, \chi$ and $\delta$ are as before, but now $A_{m}$ and $B_{m}$ are the solutions to the system of equations

$$
\begin{aligned}
& A_{m}-C_{m} \sum_{n=1}^{\infty}\left(\frac{A_{n}}{\alpha_{m}+\alpha_{n}}+\frac{B_{n}}{\alpha_{m}+\beta_{n}}\right)=-C_{m} \\
& B_{m}-D_{m} \sum_{n=1}^{\infty}\left(\frac{A_{n}}{\beta_{m}+\alpha_{n}}+\frac{B_{n}}{\beta_{m}+\beta_{n}}\right)=-D_{m}
\end{aligned}
$$

with $m=1,2, \ldots$

In Figure 3 a typical set of trapped-mode wavenumbers, $k d / \pi$, is plotted against $a / d$ for $b / d=0.6$. The solid lines correspond to modes symmetric about $x=0$ and the dashed lines correspond to modes antisymmetric about $x=0$. As $a / d$ increases the modes appear alternately symmetric and antisymmetric from the cut-off $k d=\pi / 2$ and decrease towards $k d=\pi d / 2(d+b)=0.3125 \pi$ as $a / d$ increases.

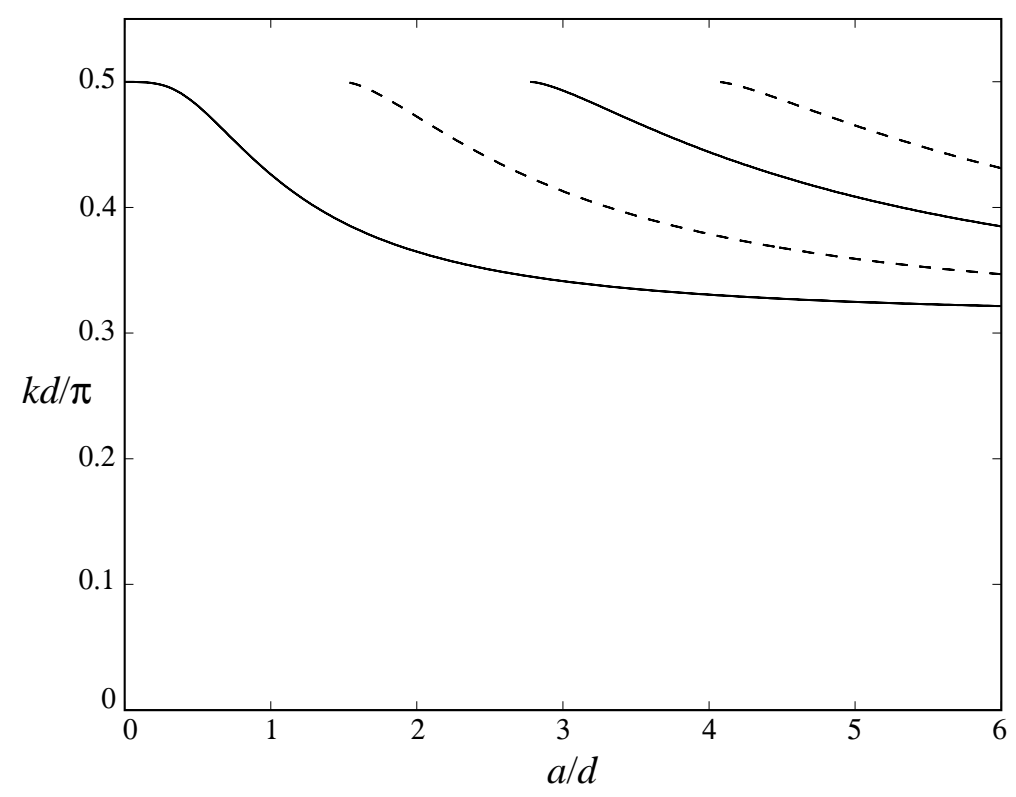

Figure 3: Trapped-mode wavenumbers $k d / \pi$ for modes symmetric $(-)$ and antisymmetric $(--)$ about $x=0$ plotted against $a / d$ when $b / d=0.6$.

The variation of trapped-mode wavenumbers with $b / d$ when $a / d=6$ is illustrated in Figure 4. Again the solid lines represent modes symmetric about $x=0$ and the dashed lines correspond to modes antisymmetric about $x=0$. The number of modes that exist can be seen to increase as $b / d$ increases from 0 . Note that there are no modes when $b=0$, which follows from the remarks preceding (2.1). It is noteworthy, however, that modes exist for all $b>0$, although for shapes of non-zero area the variational proof above only establishes existence for $b / d>1 / 2$.

\section{$3 \quad$ Neumann modes $k d<\pi / 2$}

In this section we consider the Neumann problem with $k<\lambda_{1}$. With this condition one mode can propagate down the guide, namely waves of the form $\exp \left( \pm \gamma_{0} x\right)=\exp ( \pm \mathrm{i} k x)$. The case of a shape $B$ which is symmetric about $y=0$ is well understood and a general 


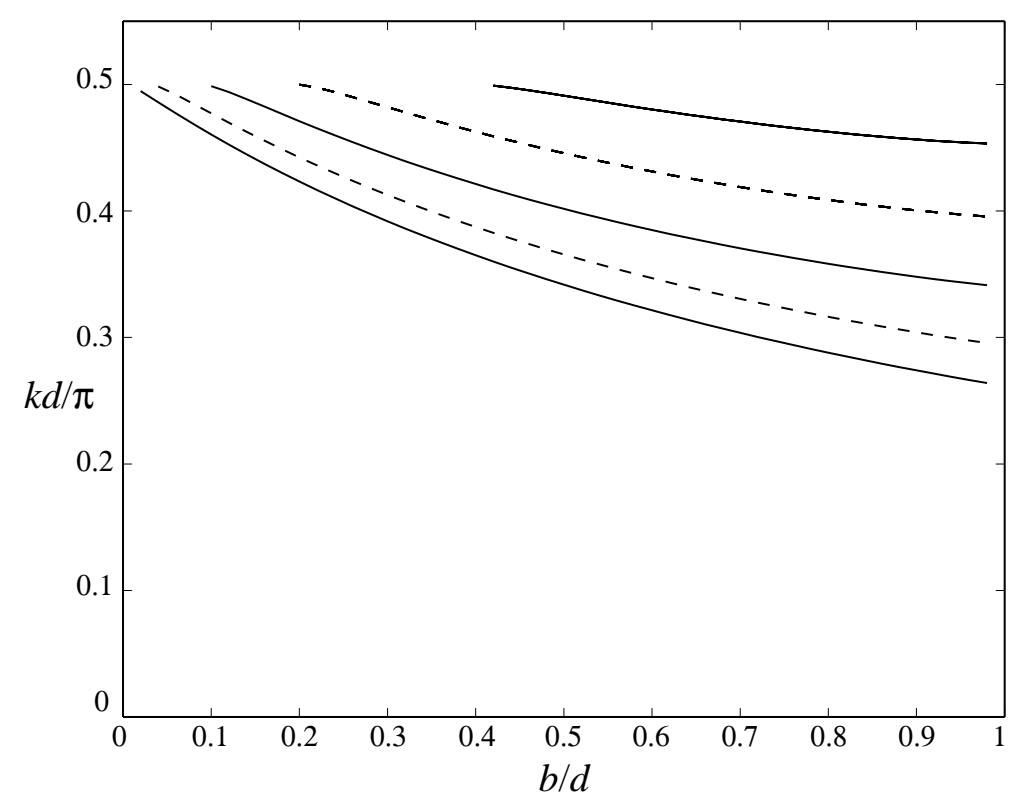

Figure 4: Trapped-mode wavenumbers $k d / \pi$ for modes symmetric $(-)$ and antisymmetric $(--)$ about $x=0$ plotted against $b / d$ when $a / d=6$.

existence result was established by Evans et al. (1994) using the symmetry of the geometry to decompose the problem into its symmetric and antisymmetric parts. Another case which has been treated is that of a thin plate aligned with the guide walls. Evans et al. (1993) solved this problem using residue calculus theory and proved the existence of modes for sufficiently long plates and it was subsequently shown that this was a special case of a more general class of modes which exist in cylindrical guides in higher dimensions (Groves 1998, Linton and McIver 1998, Davies and Parnovski 1998). The property that the thin plate has which makes it possible to consider it as a special case is that an incident wave of the form $\phi=\exp ( \pm \mathrm{i} k x)$ is not scattered by the plate.

Here we will consider shapes which do not have either of the simplifying properties mentioned above and we will refer to any modes that are found as embedded modes, in view of the fact that we are unable to decompose the problem so that they correspond to eigenvalues below the continuous spectrum of some differential operator.

\section{Numerical computations}

Numerical calculations, performed using the boundary integral equation method described in McIver et al. (2001) (in which a homogeneous integral equation of the second kind is solved subject to the satisfaction of a side condition) are presented below for a family of shapes whose boundaries are described parametrically by

$$
x=a(1-2 \delta) \cos \theta, \quad y=b+a(1+\delta \operatorname{sgn} \theta) \sin \theta, \quad \theta \in[-\pi, \pi) .
$$

The parameter $a$ is a measure of the size of the obstacle, we call $b$ the offset value, and $\delta$ governs the shape, with $\delta=0$ corresponding to a circle, see Figure 5.

The results of the numerical calculations are shown by the solid curves in Figure 6, in which the dashed curves represent the results from the slender-body theory presented below. Results are given for three different values of $a / d$, namely $0.25,0.5$, and 0.75 . For 


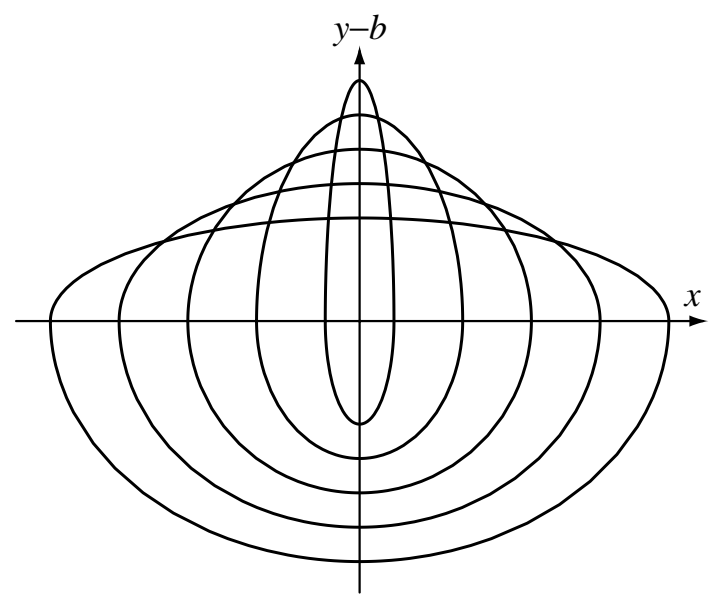

Figure 5: Shapes defined by (3.1) for various values of $\delta$. Starting from the bottom, the curves correspond to $\delta=-0.4,-0.2,0,0.2$, and 0.4 .

each value of $a / d$ we find pairs of values $(b / d, k d / \pi)$ at which embedded trapped modes exist. The shapes defined by (3.1) become more slender as $\delta$ approaches 0.5 , at which point we would have a thin plate perpendicular to the guide walls, and the increased accuracy of the slender-body theory in predicting both the offset and $k d$ values in this limit is clear.

For the case of a shape $B$ which is symmetric about $y=0$, Maniar and Newman (1997) identified the existence of trapped modes with the occurrence of unusually large forces on bottom-mounted vertical cylinders having cross-section $\partial B$, situated in the centre of a large row of such cylinders and subject to an incident surface water-wave parallel to the row. (For such problems the depth dependence can be factored out, resulting in the two-dimensional Helmholtz equation.) Calculations, using the panel code WAMIT developed at MIT, show that the same phenomenon occurs for the embedded modes shown in Figure 6.

As an example, consider the shapes defined by (3.1) with $a / d=0.5$ and $\delta=-0.2$. For such a shape, computations predict a trapped mode when $b / d \approx 0.111$ and $k d \approx 1.265$ $(k d / \pi \approx 0.403)$. An infinite array of such shapes can be constructed so that the lines of symmetry of the array correspond to the guide walls as in Figure 1, with each shape having the appropriate offset predicted by the trapped mode calculations. A section of such an array (rotated through $90^{\circ}$ ) is shown in Figure 7.

The scattering of an incident surface water wave by a finite array of bottom-mounted vertical cylinders, whose cross-sections form part of the infinite array described above, can then be considered. Specifically, we take 20 cylinders and assume that the incident wave is parallel to the cylinder row (from the left, say, in Figure 7). After the depth dependence has been factored out, the problem reduces to solving the Helmholtz equation in the region outside the cylinders, with the wavenumber $k$ being related to the frequency $\omega$ through the dispersion relation $k \tanh k h=\omega^{2} / g$, where $h$ is the water depth and $g$ is the acceleration due to gravity.

The solid curve in Figure 8 shows the magnitude of the wave load (non-dimensionalized with respect to $\rho g A a h ; \rho$ being the water density and $A$ the incident wave amplitude) on 
a)

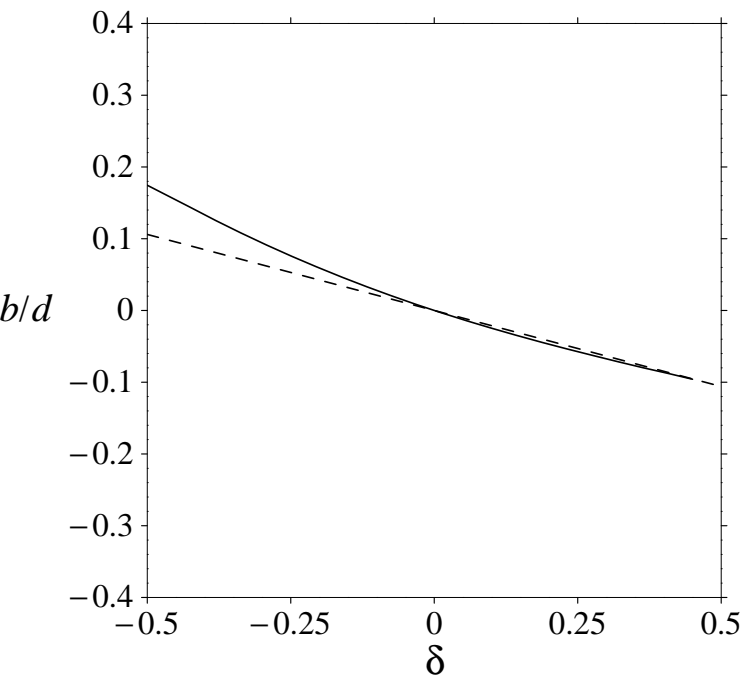

b)

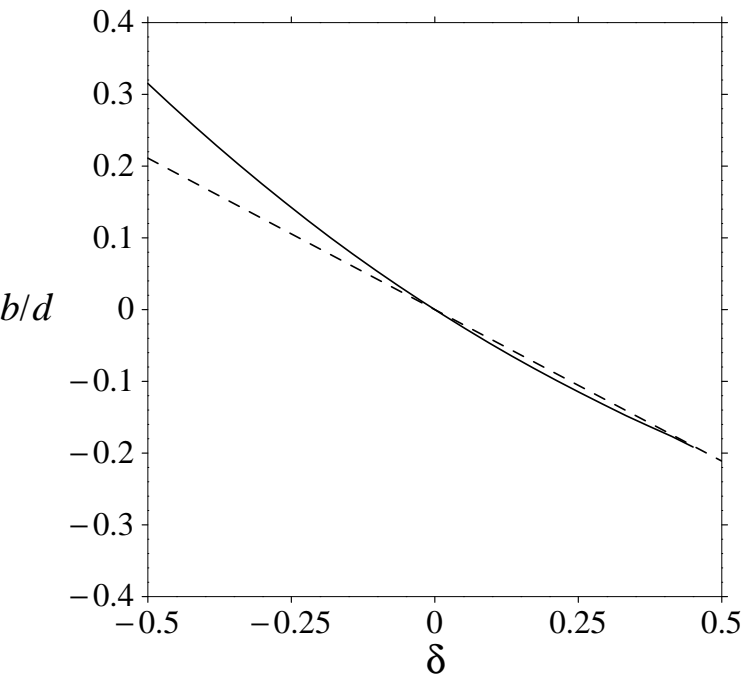

c)

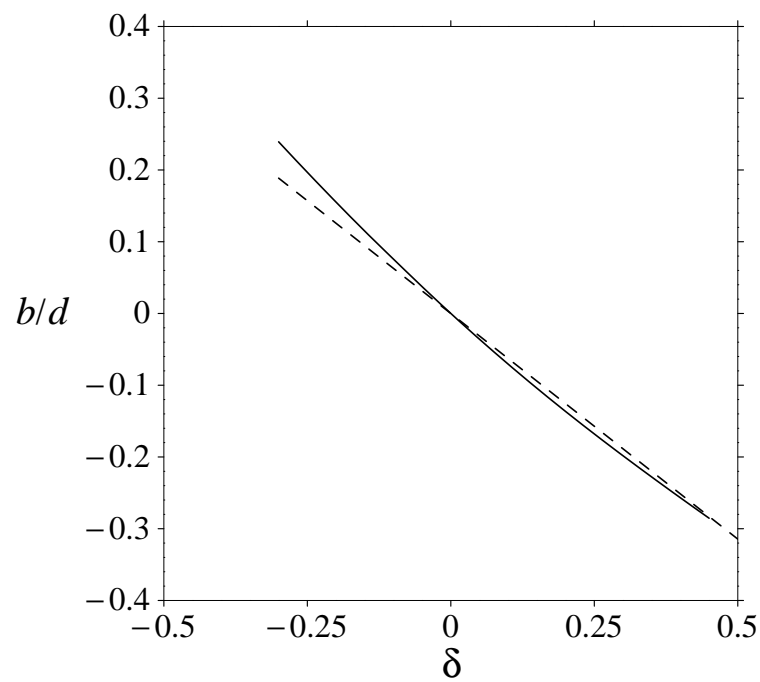

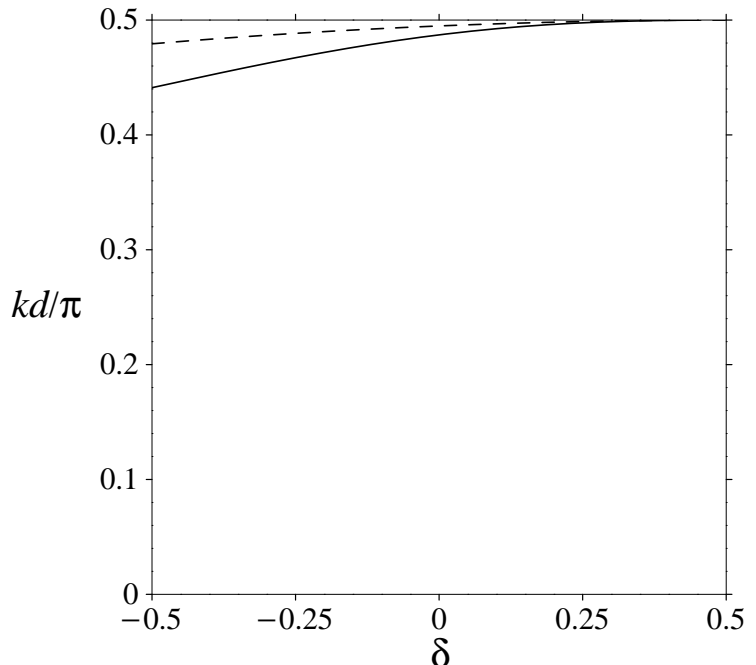
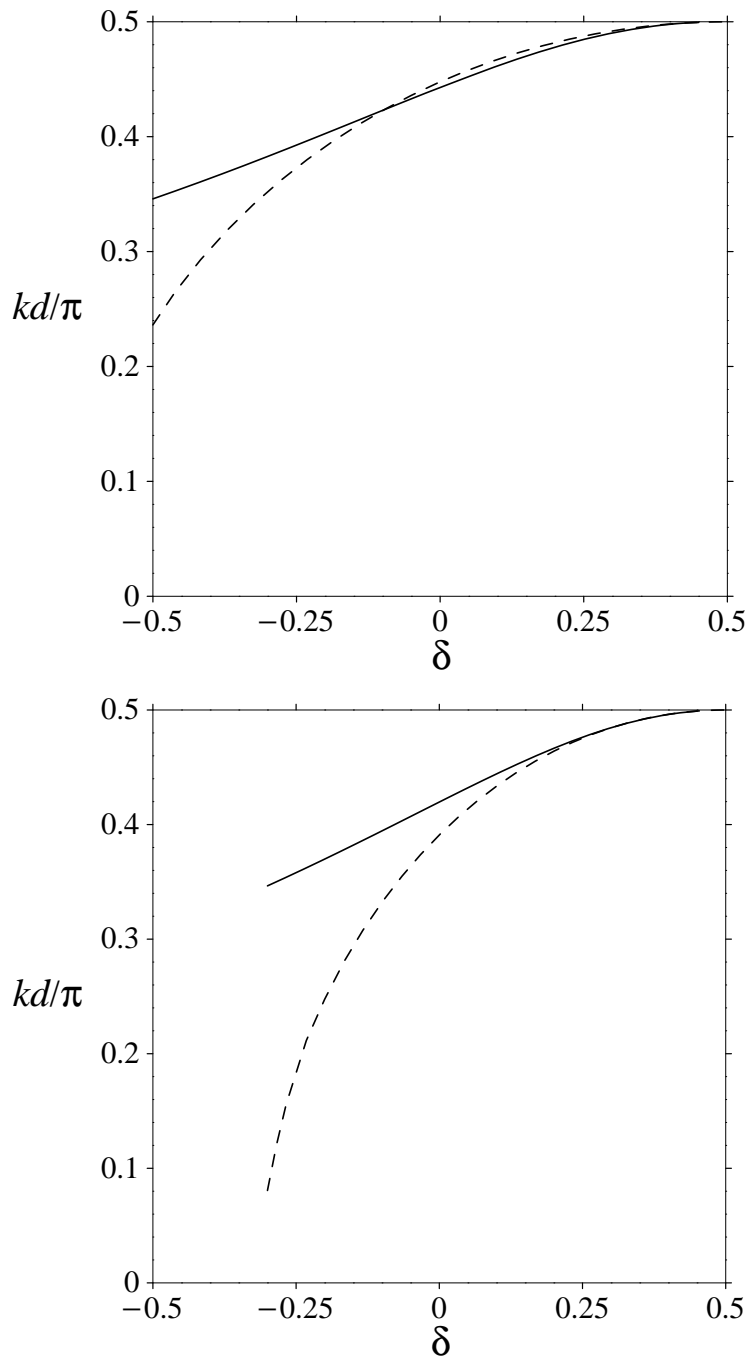

Figure 6: Offset values and trapped-mode wavenumbers for shapes defined by (3.1). Solid curves correspond to numerical computations and dashed curves to the slender-body approximation. a) $a / d=0.25$, b) $a / d=0.5$, c) $a / d=0.75$. 


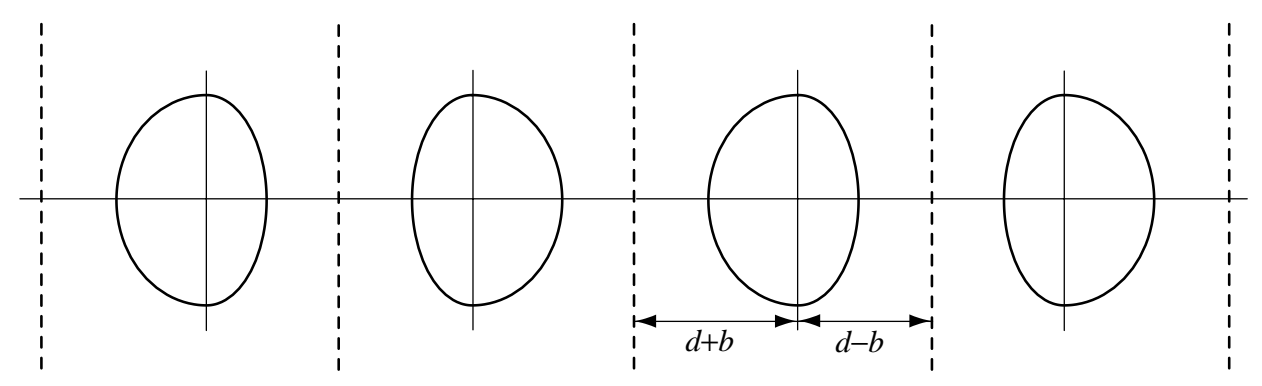

Figure 7: Shapes defined by (3.1), with $a / d=0.5, b / d=0.111$, and $\delta=-0.2$, arranged so as to form part of an infinite array. The dashed lines represent lines of symmetry.

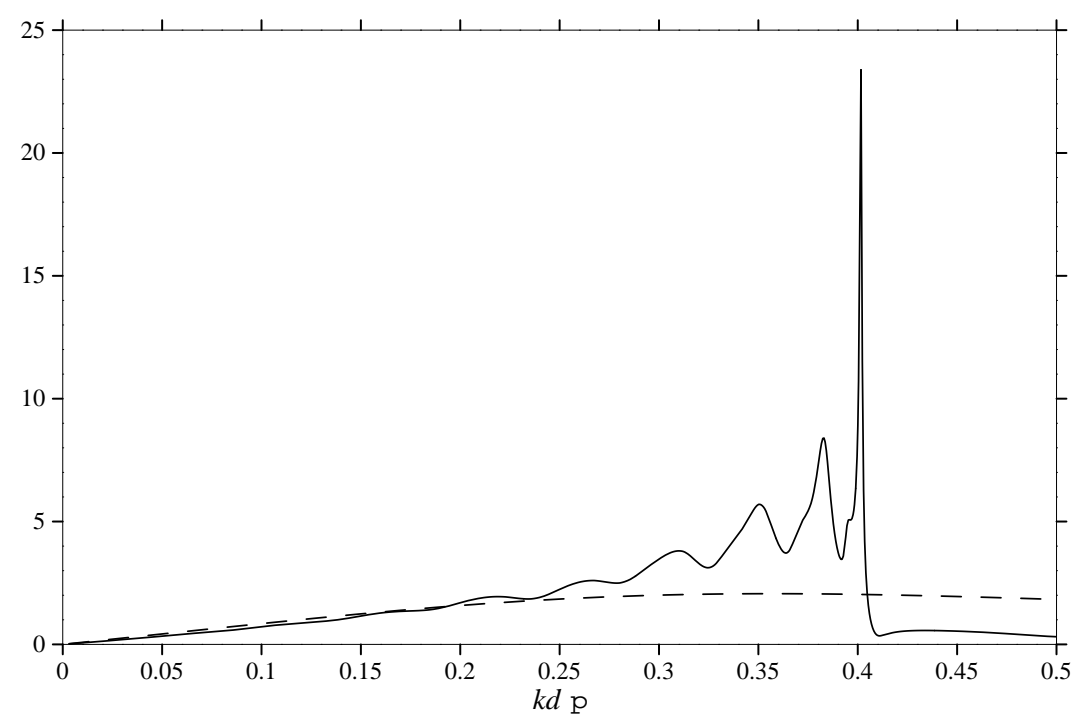

Figure 8: Wave loads on a vertical cylinder whose cross-section is defined by (3.1), with $a / d=0.5$, plotted against $k d / \pi$. The solid line is for a cylinder in the middle of a row of 20 , as described in the text, with the wave direction parallel to the row, and the dashed line is for a cylinder in isolation.

one of the two central cylinders in the array. For comparison, the dashed line shows the load that the cylinder would experience if it were in isolation, and it is clear that near the predicted trapped-mode wavenumber there is a sharp peak, resulting in wave loads about 12 times the open-sea values. Convergence tests suggest that over the bulk of the wavenumber range shown, the results are accurate to well within $1 \%$, but near the sharp resonance peak there may be errors of a few percent.

\section{Slender shapes}

The equation corresponding to (2.10) for the Neumann case is

$$
\gamma_{1}=-\frac{\epsilon \pi}{4 d^{2}} \int_{-d}^{d} f^{\prime}(y) \sin 2 \lambda_{1} y \mathrm{~d} y
$$


but now the possible existence of waves of the form $\exp (\mathrm{i} k x)$ leads to the need to satisfy the side condition

$$
\int_{-d}^{d} f^{\prime}(y) \cos \lambda_{1} y \mathrm{~d} y=0
$$

which ensures that the amplitude of any waves is zero. If the obstacle is symmetric about $y=0$ then (3.3) is satisfied identically and (3.2) reduces to equation (4.5) in Evans and McIver (1991).

Consider a shape which is symmetric about $y=b$ and is defined by a function $f(y)$ which is zero if $y \notin(b-c, b+c),(|b \pm c|<d)$. Suppose further that $f^{\prime}(y)$ is one signed for $y \in(b, b+c)$; an off-centre ellipse is an example of such an obstacle. For such obstacles the existence condition (3.3) can be satisfied only if $b=0$ so that the problem reduces to the symmetric (about $y=0$ ) case investigated by Evans et al. (1994) and others. Hence, for this class of symmetric obstacles there would appear to be no embedded trapped modes for $k d \in(0, \pi / 2)$.

If the obstacle does not have a line of symmetry parallel to the $x$-axis the existence condition can be satisfied, and hence an embedded trapped mode found, for many shape functions. For shapes given by (3.1), the existence condition reduces to

$$
\int_{-\pi / 2}^{\pi / 2} \sin \theta \cos \left[\lambda_{1}(b+a(1+\delta \operatorname{sgn} \theta) \sin \theta)\right] \mathrm{d} \theta=0
$$

solutions to which can easily be found numerically, and then the trapped-mode wavenumber can be found from

$$
\gamma_{1}=\frac{\pi a}{4 d^{2}}(1-2 \delta) \int_{-\pi / 2}^{\pi / 2} \sin \theta \sin \left[\lambda_{1}(b+a(1+\delta \operatorname{sgn} \theta) \sin \theta)\right] \mathrm{d} \theta .
$$

For a given value of $a / d$, the slender-body theory predicts the existence of modes for pairs $(b / d, k d / \pi)$, just as the numerical computations do. For $\delta>0$, which corresponds to shapes whose extension in the $y$-direction is greater than in the $x$-direction, the results from slender-body theory are quite accurate, whereas as $\delta$ decreases from zero the accuracy of the results also decreases. Because a circle corresponding to $\delta=0$ is symmetric about $y=b$, the slender-body approximation predicts the correct offset $(b=0)$ for that case.

Another example, for which the integrals can be evaluated explicitly, is a triangular obstacle described by

$$
x=\epsilon f(y) \equiv \frac{a}{c}(y-b-c)[H(y-b-c)-H(y-b)], \quad y \in[-d, d],
$$

where $H$ denotes the Heaviside step function, the base of the triangle is at $y=b$, and $c$ is the height of the triangle. In this case, the existence condition reduces to

$$
\tan \lambda_{1} b=\frac{\lambda_{1} c-\sin \lambda_{1} c}{\cos \lambda_{1} c-1}
$$

and for a sufficiently small $c / d>0$ there is one solution for $b \in(-d, 0)$ so that the obstacle straddles $y=0$ (if $c / d$ is chosen to be too large the obstacle extends outside the guide). As $c / d \rightarrow 0, b \sim-c / 3$ and the trapped mode frequency satisfies

$$
\gamma_{1} \sim \pi^{2} a c / 8 d^{3}
$$




\section{Dirichlet modes $\pi / 2<k d<\pi$}

In this section we consider the Dirichlet problem with $\lambda_{1}<k<\lambda_{2}$ for slender shapes. As in the previous section this condition allows for one propagating mode down the guide, in this case corresponding to waves of the form

$$
\sin \lambda_{1}(y+2 d) \mathrm{e}^{ \pm \gamma_{1} x} .
$$

The equation for the trapped mode frequencies is now

$$
\gamma_{2}=-\frac{\epsilon \pi}{2 d^{2}} \int_{-d}^{d} f^{\prime}(y) \sin 2 \lambda_{2} y \mathrm{~d} y
$$

and the side condition which needs to be satisfied is

$$
\int_{-d}^{d} f^{\prime}(y) \cos \lambda_{1} y \cos \lambda_{2} y \mathrm{~d} y=0
$$

An obstacle that is symmetric about $y=0$ with a solution that is antisymmetric about $y=0$, corresponds to an asymmetric structure within a Dirichlet guide of width $d$. Equation (4.2) is then satisfied identically and (4.1) becomes

$$
\gamma_{2}=-\frac{\pi \epsilon}{d^{2}} \int_{0}^{d} f^{\prime}(y) \sin 2 \lambda_{2} y \mathrm{~d} y
$$

which, after adjustment of the guide width and origin of coordinates, may be seen to be exactly equivalent to (2.10).

For the triangle given by (3.6) the existence condition (4.2) has real solutions such that as $c / d \rightarrow 0$ then $b \sim-c / 3$. It then follows from (4.1) that

$$
\gamma_{2} \sim \pi^{2} a c / 2 d^{3}
$$

For a semicircle of radius $a$ with its flat edge on $y=d$ the existence condition (4.2) is satisfied for $a / d \approx 0.59$. This situation is equivalent to one studied by Evans and Porter (1998) for which a value of $a / d \approx 0.53$ was obtained using a multipole method.

\section{$5 \quad$ Neumann modes $\pi<k d<3 \pi / 2$}

The existence of trapped modes in the cases considered in $\S \S 3-4$ involve the satisfaction of a side condition which corresponds to ensuring that the amplitude of any propagating mode is zero. In each case there is one propagating mode and so one side condition. Here we will examine a situation where two side conditions are involved. Specifically we will look at a plate aligned with the guide walls (i.e. $B$ is $\{(x, y): y=b, x \in[-a, a]$ ) on which Neumann conditions are applied and restrict attention to the frequency range $\lambda_{2}<k<\lambda_{3}$. In this range three types of propagating mode are possible in the main body of the guide:

$$
\mathrm{e}^{ \pm \mathrm{i} k x}, \quad \mathrm{e}^{ \pm \mathrm{i} \gamma_{1} x} \cos \lambda_{1}(y+d) \quad \text { and } \quad \mathrm{e}^{ \pm \mathrm{i} \gamma_{2} x} \cos \lambda_{2}(y+d)
$$

For the modes above and below the plate we define

$$
\begin{gathered}
\nu_{n}=n \pi /(d-b), \quad \alpha_{n}=\left(\nu_{n}^{2}-k^{2}\right)^{1 / 2}=-\mathrm{i}\left(k^{2}-\nu_{n}^{2}\right)^{1 / 2} \\
\mu_{n}=n \pi /(d+b), \quad \beta_{n}=\left(\mu_{n}^{2}-k^{2}\right)^{1 / 2}=-\mathrm{i}\left(k^{2}-\mu_{n}^{2}\right)^{1 / 2}
\end{gathered}
$$


and provided

$$
\frac{\pi d}{d-b}<k d<\frac{3 \pi}{2}
$$

for which we require $b / d<1 / 3$, it follows that $\alpha_{0}=\beta_{0}=-\mathrm{i} k, \alpha_{1}=-\mathrm{i} \alpha$ and $\beta_{1}=-\mathrm{i} \beta$, where $\alpha$ and $\beta$ are real and positive, all the other $\alpha$ 's and $\beta$ 's being real. In other words, provided (5.3) is satisfied, two modes are possible in the regions above and below the plate:

$$
\begin{aligned}
& \mathrm{e}^{ \pm \mathrm{i} k x} \text { and } \mathrm{e}^{ \pm \mathrm{i} \alpha x} \cos \nu_{1}(d-y) \text { in } b<y<d, \\
& \mathrm{e}^{ \pm \mathrm{i} k x} \text { and } \mathrm{e}^{ \pm \mathrm{i} \beta x} \cos \mu_{1}(d+y) \text { in }-d<y<b \text {. }
\end{aligned}
$$

This turns out to be precisely the right number of propagating modes which enables us to construct solutions which decay as $|x| \rightarrow \infty$.

The method that we use to construct trapped modes is the modified residue calculus theory which was used for the Neumann problem and the same geometry (with $k d<\pi / 2$ ) by Evans et al. (1993). The procedure follows closely that given in their paper and only brief details illustrating the main differences will be given here. If we seek trapped modes which are symmetric about $x=0$, then matching eigenfunction expansions in the regions above and below the plate with an expansion valid for $x>a$ leads to the systems of equations

$$
\begin{aligned}
& \sum_{n=3}^{\infty} U_{n}\left\{\frac{1}{\gamma_{n}-\alpha_{m}}+\frac{\mathrm{e}^{-2 \alpha_{m} a}}{\gamma_{n}+\alpha_{m}}\right\}=0, \quad m=0,1,2, \ldots, \\
& \sum_{n=3}^{\infty} U_{n}\left\{\frac{1}{\gamma_{n}-\beta_{m}}+\frac{\mathrm{e}^{-2 \beta_{m} a}}{\gamma_{n}+\beta_{m}}\right\}=0, \quad m=0,1,2, \ldots,
\end{aligned}
$$

for some unknown coefficients $U_{n}$, equations which are identical to those in Evans et al. (1993) except that in their paper the summations start from one. In the present situation the summations start from three because we require the three possible modes as $x \rightarrow \infty$ to have zero amplitude, corresponding to $U_{0}=U_{1}=U_{2}=0$.

Information about the solution to these equations can be obtained by consideration of the function

$$
f(z)=g(z) h(z)
$$

where

$$
\begin{aligned}
& g(z)=\mathrm{e}^{z \Theta}\left(1-z / \alpha_{2}\right)\left(1-z / \beta_{2}\right) \prod_{n=3}^{\infty} \frac{\left(1-z / \alpha_{n}\right)\left(1-z / \beta_{n}\right)}{\left(1-z / \gamma_{n}\right)}, \\
& h(z)=1+\sum_{n=2}^{\infty}\left(\frac{A_{n}}{z-\alpha_{n}}+\frac{B_{n}}{z-\beta_{n}}\right),
\end{aligned}
$$

in which $\Theta$ is defined in $(2.21)$ and $A_{n}$ and $B_{n}$ are undetermined. The function $g(z)$ contains a factor for each of the numbers $\alpha_{n}, \beta_{n}$ and $\gamma_{n}$ that are real and it can be shown that $f(z)=O\left(z^{-1 / 2}\right)$ as $z \rightarrow \infty$ avoiding certain real positive values. This result is crucial to the success of the residue calculus method.

The solution to (5.4) and (5.5) is given by

$$
U_{n}=\mathrm{R}\left(f: \gamma_{n}\right)
$$


where $\mathrm{R}\left(f: z_{0}\right)$ is the residue of $f(z)$ at $z=z_{0}$, provided we also satisfy the conditions

$$
\begin{aligned}
& f\left(\alpha_{m}\right)+\mathrm{e}^{-2 \alpha_{m} a} f\left(-\alpha_{m}\right)=0, \quad m=0,1,2, \ldots, \\
& f\left(\beta_{m}\right)+\mathrm{e}^{-2 \beta_{m} a} f\left(-\beta_{m}\right)=0, \quad m=0,1,2, \ldots .
\end{aligned}
$$

For $m \geq 2$ these conditions lead to an exponentially convergent infinite system of real equations for the unknowns $A_{n}$ and $B_{n}$, similar in form to (2.22) and (2.23), while for $m=0$ and 1 we obtain

$$
\begin{gathered}
f(-\mathrm{i} k)+\mathrm{e}^{2 \mathrm{i} k a} f(\mathrm{i} k)=0, \\
f(-\mathrm{i} \alpha)+\mathrm{e}^{2 \mathrm{i} \alpha a} f(\mathrm{i} \alpha)=0 \\
f(-\mathrm{i} \beta)+\mathrm{e}^{2 \mathrm{i} \beta a} f(\mathrm{i} \beta)=0 .
\end{gathered}
$$

If we define

$$
\chi(x)=\tan ^{-1} \frac{x}{\alpha_{2}}+\tan ^{-1} \frac{x}{\beta_{2}}+\sum_{n=3}^{\infty}\left(\tan ^{-1} \frac{x}{\alpha_{n}}+\tan ^{-1} \frac{x}{\beta_{n}}-\tan ^{-1} \frac{x}{\gamma_{n}}\right)
$$

and

$$
\delta(x)=\arg \left(1-\sum_{n=2}^{\infty}\left[\frac{A_{n}}{\alpha_{n}+\mathrm{i} x}+\frac{B_{n}}{\beta_{n}+\mathrm{i} x}\right]\right),
$$

then (5.12)-(5.14) reduce to

$$
\begin{aligned}
& k(a+\Theta)=\left(l+\frac{1}{2}\right) \pi+\chi(k)+\delta(k), \\
& \alpha(a+\Theta)=\left(m+\frac{1}{2}\right) \pi+\chi(\alpha)+\delta(\alpha), \\
& \beta(a+\Theta)=\left(n+\frac{1}{2}\right) \pi+\chi(\beta)+\delta(\beta),
\end{aligned}
$$

for some integers $l, m, n$.

The first observation to make about these conditions is that as $a / d \rightarrow \infty, \delta(x)$ tends rapidly to zero. As we will show below, trapped modes only exist for values of $a / d>1$ and all of the values computed are such that the $\delta$ terms in (5.17)-(5.19) are numerically insignificant. Hence we will ignore them from now on. This is the great advantage of the modified residue calculus method; for all but the smallest values of $a / d$, the solution can be determined from explicit formulas, rather than requiring the inversion of a system of equations.

A very crude, but as it turns out quite accurate, approximation to the solution to our problem can be found by simply assuming that the potential in the region $x>a$ is identically zero. Then in the regions $0<x<a, b<y<d$ and $0<x<a, 0<y<b$ we can assume that $\phi$ takes the form

$$
A \cos (2 l+1) \frac{\pi x}{2 a}+B \cos (2 m+1) \frac{\pi x}{2 a} \cos \frac{\pi(d-y)}{d-b}
$$

and

$$
A \cos (2 l+1) \frac{\pi x}{2 a}+B \cos (2 n+1) \frac{\pi x}{2 a} \cos \frac{\pi(d+y)}{d+b},
$$

respectively, where $l, m, n$ are non-negative integers. (Of course, for such a solution $\phi_{x}$ is not continuous across $x=a$.) For each term in the above expressions to satisfy the Helmholtz equation for the same value of $k$ we would require

$$
k^{2}=\left(l+\frac{1}{2}\right)^{2} \frac{\pi^{2}}{a^{2}}=\left(m+\frac{1}{2}\right)^{2} \frac{\pi^{2}}{a^{2}}+\frac{\pi^{2}}{(d-b)^{2}}=\left(n+\frac{1}{2}\right)^{2} \frac{\pi^{2}}{a^{2}}+\frac{\pi^{2}}{(d+b)^{2}} .
$$


Equivalently

$$
k a=\left(l+\frac{1}{2}\right) \pi, \quad \alpha a=\left(m+\frac{1}{2}\right) \pi, \quad \beta a=\left(n+\frac{1}{2}\right) \pi,
$$

which is just (5.17)-(5.19) without $\Theta, \chi$ or $\delta$. Eliminating $k$ and $a$ from (5.20), we find that

$$
\frac{(2 l+1)^{2}-(2 m+1)^{2}}{(2 l+1)^{2}-(2 n+1)^{2}}=\left(\frac{d+b}{d-b}\right)^{2}>1,
$$

from which it follows that a necessary condition that $0<b / d<1 / 3$ is

$$
l>n \geq m \geq 0 .
$$

Thus, for any given triple of integers satisfying (5.23), we can calculate $b / d$ from (5.22) and, if $b / d<1 / 3, k d$ and $a / d$ follow easily from (5.20). We might therefore hope that solutions to (5.17)-(5.19), in which the terms $\Theta, \chi$ and $\delta$ are all ignored, are good approximations to the full problem. That this is the case is illustrated in Figure 9 in which the dots have been calculated by solving (5.21), whereas the crosses are solutions to (5.17)-(5.19) with $\Theta$ and $\chi$ included. It is also possible to construct an approximate solution which satisfies continuity of $\phi_{x}$ at $x=a$, but not continuity of $\phi$. This leads to a condition of the form (5.22) but with $2 l+1,2 m+1,2 n+1$ replaced by $2 l, 2 m$, and $2 n$ respectively. The resulting numerical approximations to the triples $(a / d, b / d, k d)$ at which trapped modes exist are not as good as those shown in the figure.

Equation (5.17) shows that, since we are only considering values of $k d$ in the range $(\pi, 3 \pi / 2)$, the maximum possible value of $a / d$ is governed by the magnitude of the integer $l$. In the results shown in Figure 9, all integer triples satisfying (5.23) with $l \leq 14$ were used (for which $b / d<1 / 3$ and $k d \in(\pi, 3 \pi / 2)$ ) and this ensures that all eigenvalues with $a / d<10$ have been found. Thus the two uppermost panels in the figure are complete, whereas there are more eigenvalues that would appear in the lowest panel corresponding to larger values of $l$.

\section{Conclusion}

The question of the existence of trapped modes near obstacles in two-dimensional waveguides has been considered for cases where there is no symmetry about the centre-line of the guide. The boundary condition on the obstacle is of Neumann type and both Neumann and Dirichlet conditions on the guide walls have been examined.

For the Dirichlet problem below the cut-off for propagating modes we have shown that trapped modes do exist for a class of obstacles, although it is known that for other classes they do not. In general, the closer a body is to the centreline, the less likely it is for a trapped mode to exist. An exception to this is a thin plate aligned with the guide walls, for which modes exist for any offset greater than zero.

For the Dirichlet problem between the first and second cut-offs and for the Neumann problem below the first cut-off (situations in which one propagating mode is possible in the guide) trapped modes are also possible, though in these cases we need to consider families of geometries defined by two parameters. The modes then exist if these parameters are related in a certain way.

Finally for the Neumann problem between the second and third cut-offs, so that three propagating modes are possible in the guide, we have solved the problem for a specific geometry (a thin plate aligned with the guide walls) and shown that modes exist for discrete pairs of the two parameters which define the geometry. 

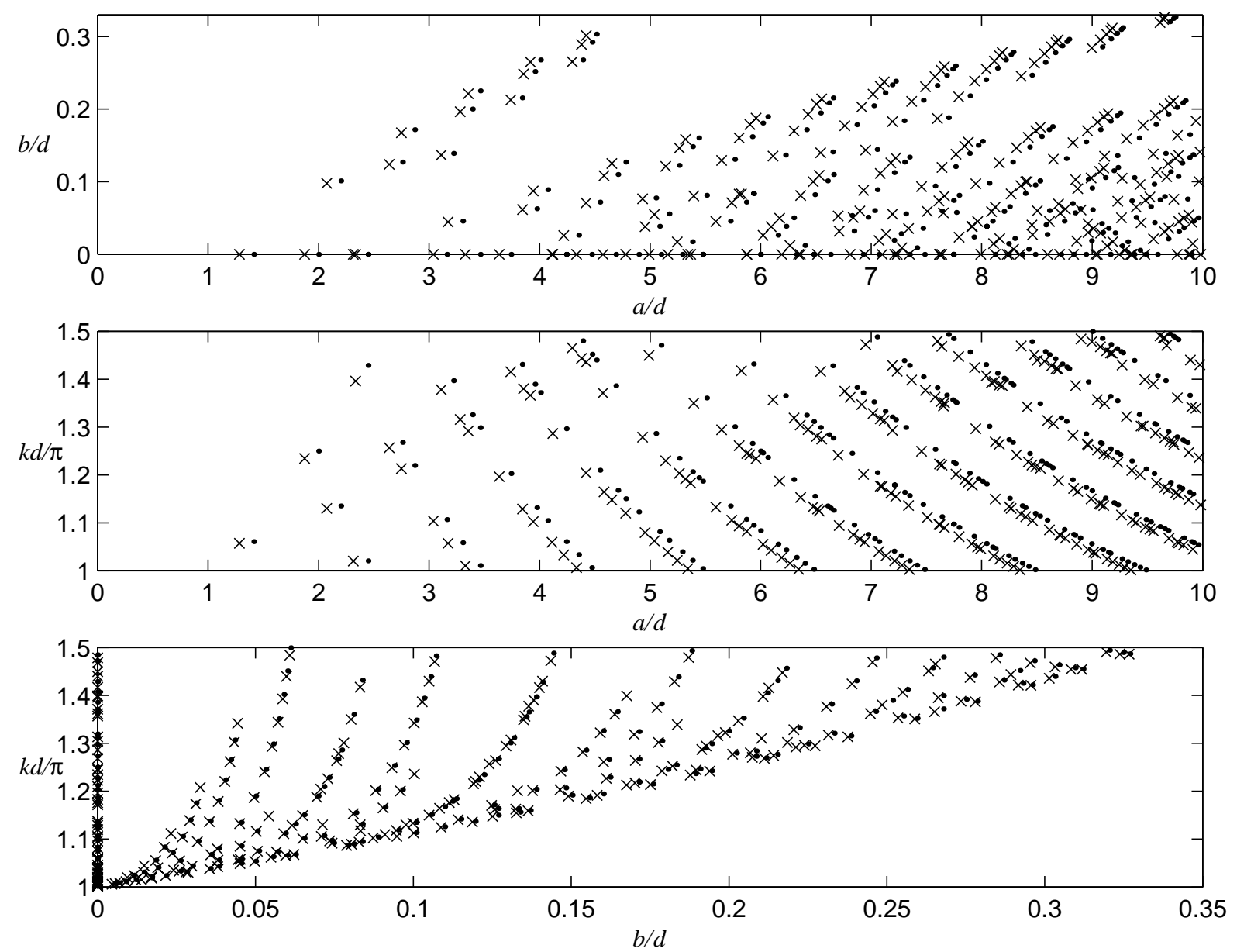

Figure 9: Triples $(a / d, b / d, k d)$ corresponding to embedded trapped modes for an offcentre plate. The dots are the solutions to (5.21) while the crosses are found from (5.17)$(5.19)$

\section{Acknowledgements}

The work of JZ was supported by EPSRC grant GR/M30937. The work of KR was supported by an EPSRC studentship.

\section{References}

Davies, E. B., \& Parnovski, L. (1998). Trapped modes in acoustic waveguides. Q. J. Mech. Appl. Math., 51, 477-492.

Evans, D. V., Levitin, M., \& Vassiliev, D. (1994). Existence theorems for trapped modes. J. Fluid Mech., 261, 21-31.

Evans, D. V., Linton, C. M., \& Ursell, F. (1993). Trapped mode frequencies embedded in the continuous spectrum. Q. J. Mech. Appl. Math., 46(2), 253-274.

Evans, D. V., \& McIver, P. (1991). Trapped waves over symmetric thin bodies. J. Fluid Mech., 223, 509-519. 
Evans, D. V., \& Porter, R. (1998). Trapped modes embedded in the continuous spectrum. Q. J. Mech. Appl. Math., 51(2), 263-274.

Groves, M. D. (1998). Examples of embedded eigenvalues for problems in acoustic waveguides. Math. Meth. in the Appl. Sci., 21, 479-488.

Linton, C. M., \& McIver, M. (1998). Trapped modes in cylindrical guides. Q. J. Mech. Appl. Math., 51, 389-412.

Maniar, H. D., \& Newman, J. N. (1997). Wave diffraction by a long array of cylinders. J. Fluid Mech., 339, 309-330.

McIver, M., Linton, C.M., McIver, P., Zhang, J., \& Porter, R. (2001). Embedded trapped modes for obstacles in two-dimensional waveguides. To appear in $Q$. J. Mech. Appl. Math.

McIver, M., \& Linton, C. M. (1995). On the non-existence of trapped modes in acoustic waveguides. Q. J. Mech. Appl. Math., 48(4), 543-555.

McIver, P. (2001). Approximations to embedded trapped modes in wave guides. In Proc. of IUTAM Conference 2000/10 on Diffraction and Scattering in Fluid Mechanics and Elasticity, Manchester, UK.

Porter, R., \& Evans, D. V. (1999). Rayleigh-Bloch surface waves along periodic gratings and their connection with trapped modes in waveguides. J. Fluid Mech., 386, 233-258. 